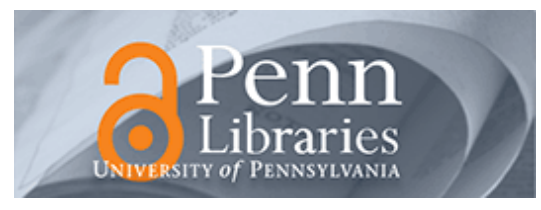

University of Pennsylvania

ScholarlyCommons

Statistics Papers

Wharton Faculty Research

$12-2010$

\title{
Maximizing Voronoi Regions of a Set of Points Enclosed in a Circle with Applications to Facility Location
}

Bhaswar B. Bhattacharya

University of Pennsylvania

Follow this and additional works at: https://repository.upenn.edu/statistics_papers

Part of the Applied Statistics Commons, Business Administration, Management, and Operations Commons, Business Analytics Commons, Management Sciences and Quantitative Methods Commons, and the Mathematics Commons

\section{Recommended Citation}

Bhattacharya, B. B. (2010). Maximizing Voronoi Regions of a Set of Points Enclosed in a Circle with Applications to Facility Location. Journal of Mathematical Modelling and Algorithms, 9 (4), 375-392. http://dx.doi.org/10.1007/s10852-010-9142-0

This paper is posted at ScholarlyCommons. https://repository.upenn.edu/statistics_papers/655

For more information, please contact repository@pobox.upenn.edu. 


\title{
Maximizing Voronoi Regions of a Set of Points Enclosed in a Circle with Applications to Facility Location
}

\author{
Abstract \\ In this paper we introduce an optimization problem which involves maximization of the area of Voronoi \\ regions of a set of points placed inside a circle. Such optimization goals arise in facility location problems \\ consisting of both mobile and stationary facilities. Let $\psi$ be a circular path through which mobile service \\ stations are plying, and $S$ be a set of $n$ stationary facilities (points) inside $\psi$. A demand point $p$ is served \\ from a mobile facility plying along $\psi$ if the distance of $p$ from the boundary of $\psi$ is less than that from any \\ member in $S$. On the other hand, the demand point $p$ is served from a stationary facility $p_{i} \in S$ if the \\ distance of $p$ from $p_{\mathrm{i}}$ is less than or equal to the distance of $p$ from all other members in $S$ and also from \\ the boundary of $\psi$. The objective is to place the stationary facilities in $S$, inside $\psi$, such that the total area \\ served by them is maximized. We consider a restricted version of this problem where the members in $S$ \\ are placed equidistantly from the center $o$ of $\psi$. It is shown that the maximum area is obtained when the \\ members in $S$ lie on the vertices of a regular $n$-gon, with its circumcenter at $o$. The distance of the \\ members in $S$ from o and the optimum area increases with $n$, and the limit approaches the radius and \\ the area of the circle $\psi$, respectively. We also consider another variation of this problem where a set of $n$ \\ points is placed inside $\psi$, and the task is to locate a new point $q$ inside $\psi$ such that the area of the Voronoi \\ region of $q$ is maximized. We give an exact solution of this problem when $n=1$ and a $(1-\varepsilon)$ - \\ approximation algorithm for the general case.

\section{Keywords} \\ computational geometry, optimization, stationary and mobile facilities, Voronoi diagrams \\ Disciplines \\ Applied Statistics | Business | Business Administration, Management, and Operations | Business Analytics \\ | Management Sciences and Quantitative Methods | Mathematics | Statistics and Probability
}




\title{
Maximizing Voronoi Regions of a Set of Points Enclosed in a Circle with Applications to Facility Location
}

\author{
Bhaswar B. Bhattacharya \\ Indian Statistical Institute, Kolkata - 700 108, India \\ bhaswar. bhattacharya@gmail.com
}

\begin{abstract}
In this paper we introduce an optimization problem, which involves maximization of the area of Voronoi regions of a set of points placed inside a circle. Such optimization goals arise in facility location problems consisting of both mobile and stationary facilities. Let $\psi$ be a circular path through which mobile service stations are plying, and $S$ be a set of $n$ stationary facilities (points) inside $\psi$. A demand point $p$ is served from a mobile facility plying along the circumference of $\psi$ if the distance of $p$ from the boundary of $\psi$ is less than that from any member in $S$. On the other hand, the demand point $p$ is served from a particular member $p_{i} \in S$ if it is closer to $p_{i}$ than from all other members in $S$ and also from the boundary of $\psi$. The objective is to place the stationary facilities in $S$, inside $\psi$, such that the total area served by them is maximized. We consider a restricted version of this problem where the members in $S$ are placed equidistantly from the center $o$ of $\psi$. It is shown that the maximum area is obtained when the members in $S$ lie on the vertices of a regular $n$-gon, with its circumcenter at $o$. The distance of the members in $S$ from $o$ and the optimum area increases with $n$, and at the limit approaches the radius and the area of the circle $\psi$, respectively. We also consider another variation of this problem where a set of $n$ points is placed inside $\psi$, and the task is to locate a new point $q$ inside $\psi$ such that the area of the Voronoi region of $q$ is maximized. We give an exact solution of this problem when $n=1$ and a $(1-\varepsilon)$-approximation algorithm for the general case.
\end{abstract}

Keywords: Computational geometry, Optimization, Stationary and mobile facilities, Voronoi diagrams.

\section{Introduction}

The main objective in any facility location problem is to judiciously place a set of facilities, serving a set of users (or demand points), such that certain optimality criteria are satisfied. Facilities can be stationary, like shops, factory outlets, hospitals, or mobile, which supply provisions to the users while on the move. The set of users, on the other hand, is either discrete, consisting of finitely many points, or continuous, i.e., a region where every point is considered to be a user. Provided all the facilities are equally equipped in all respects, a user always avails the service from its nearest facility. Thus, each facility $a$ has its service zone $Z(a)$, consisting of the set of users that are served by it. The service zone may be a finite set of points or a continuous region. Many variations of facility location problems in both the discrete and continuous category, under several optimality criteria, have been studied [12]. Maximizing the cardinality of the service zone(s) of a (type of) facility is one such criterion. In the discrete case, it generally denotes the number of users, and in the continuous case it generally represents the area served by that particular (type of) facility. In the discrete case, the problem of placing a new facility amidst existing ones, such that the number of users served by it is maximized, has been addressed very recently by Cabello et al. [8]. They proposed a general technique for solving the problem under different relevant metrics. Recently, Bhattacharya and Nandy have addressed the problem of simultaneously placing two new facilities amidst other existing facilities such that the total number of users served by the two new facilities is maximized [6]. There remain several open problems 
for continuous demand regions. Dehne et al. [11] addressed the problem of locating a new facility $p$ amidst a set of existing facilities, such that the area of the region served by the new facility is maximized.

We study two variations of a facility placement problem consisting of both mobile and stationary facilities. Here the set of facilities consists of (i) a circle $\psi$, and (ii) a set $S$ of stationary points inside $\psi$. The boundary of the circle $\psi$ represents the path of a few mobile facilities so that every point on $\psi$ is assumed to be a facility point. Our objective is to place the points in $S$ inside $\psi$ such that the total area served by the members in $S$ is maximized. We consider another variation of this problem, where the boundary of $\psi$ and an existing set $S$ is serving the region inside $\psi$; the objective is to place a new facility $q$ inside $\psi$ such that the area served by $q$ is maximized.

Both these problems are of emerging interest in the context of designing a service network for disaster management. Imagine a situation where a large locality/island is under certain disaster, e.g. flood, and the population therein needs urgent help from outside. Most of the affected region has been rendered inaccessible. In Figure 1(a), we demonstrate a situation, where a few mobile service stations are plying along a path $R$ (on roads or on rescue ships) surrounding the affected region, supplying provisions to the distressed people. A few more stationary locations (for example $p_{1}, p_{2}$ as in Figure 1(a)) are marked at some less hazardous locations, where facilities can be established, or provisions be supplied/air-dropped by the rescue team. At any point of time, a person is likely to observe an accessibility condition, i.e., he/she will only approach the nearest stationary service station, or the nearest point on the surrounding path, whichever is nearer. Since service from a mobile station cannot be obtained as soon as the user reaches its nearest point on the boundary of $\psi$, the motivation of the proposed problems is to minimize the users' dependency on the mobile stations for getting the service.

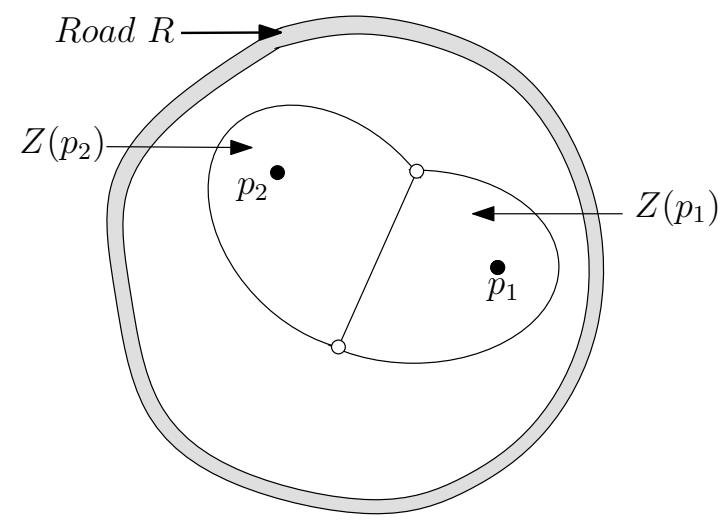

(a)

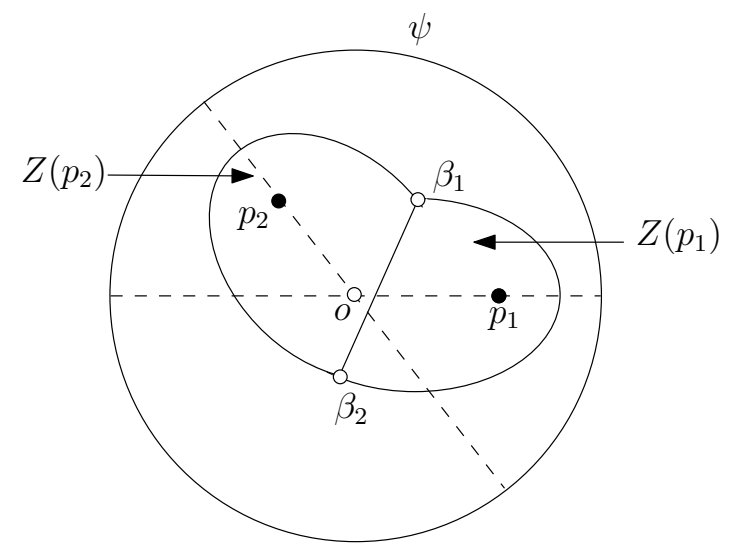

(b)

Fig. 1. Formulation of the problem

For the second problem, we again imagine a similar situation. But now apart from the circular road plying with the mobile facilities, we have some existing stationary facilities placed in the disaster area. A new and better-equipped service station is to be established amidst the existing ones and our obvious aim would be to maximize the area served by it. This problem can also be considered as an extension of the competitive facility location problems related to Voronoi games $[1,10]$. 
These two problems can be mathematically modeled as follows. Let the surrounding path $R$ be approximated by the circumference of a circle $\psi$ of radius $r$, with the center at $o$ (Figure 1(b)). It can be shown that the zone $Z\left(p_{1}\right)$ of a stationary service station $p_{1}$ inside the circle $\psi$ is an ellipse that includes the center $o$ of $\psi$. For a pair of service stations $p_{1}$ and $p_{2}$, the service zones $Z\left(p_{1}\right)$ and $Z\left(p_{2}\right)$ are no longer complete ellipses, but form elliptical sectors as shown in Figure 1(b). The first problem then reduces to maximizing the area covered by $Z\left(p_{1}\right) \cup Z\left(p_{2}\right)$, whereas the goal of the second problem is to maximize the area covered by $Z\left(p_{2}\right)$, given the position of the point $p_{1}$.

These two problems can be easily formulated in terms of maximizing the area of a Voronoi region of a set of points placed inside a circle. The Voronoi diagram of a set $S$ of $n$ points in $\mathbb{R}^{d}$, denoted by $V(S)$, is a partition of the space into $|S|$ mutually non-overlapping regions (excepting the boundaries) $\{V R(p, S) \mid p \in S\}$, where the region $V R(p, S)=Z(p)$ is the set of points in the space that are closer to the point $p$ than to any other point $q \in S$. This idea can be extended to the case when the members in $S$ are general objects instead of points $[2,15,17]$. In this paper, we consider the Voronoi diagram $V(S \cup\{\psi\})$ of the circle $\psi$ and a set $S$ of points placed inside $\psi$, under the Euclidean metric. The Voronoi region of a point $p \in S$ is denoted by $V R(p, S \cup\{\psi\})$ and that of $\psi$ is denoted by $V R(\psi, S \cup\{\psi\})$.

The optimization problems which we address can now be stated formally as follows:

P1: Given a circle $\psi$, place a set of $n$ points $S=\left\{p_{1}, p_{2}, \ldots, p_{n}\right\}$ inside $\psi$ such that Area $\left\{\bigcup_{i=1}^{n} V R\left(p_{i}, S \cup\{\psi\}\right)\right\}$ is maximized.

P2: Given a circle $\psi$ and a set of $n$ points $S=\left\{p_{1}, p_{2}, \ldots, p_{n}\right\}$ placed inside $\psi$, locate a new point $q$ inside $\psi$ such that $\operatorname{Area}\{\operatorname{VR}(q, S \cup\{\psi, q\})\}$ is maximized.

The properties, importance, and usefulness of Voronoi diagrams have been extensively studied in the literature $[4,5,7,16]$ over the past few decades. The problem of maximizing the area of Voronoi regions has been considered in the context of Hotelling game or Voronoi game $[1,10,15]$. Maximizing the area of the Voronoi region of a particular point is addressed in $[9,11]$, where the objective is to locate the position of a new point $q$ amidst a set of $n$ existing points $S$ such that the Voronoi region of $q$ is maximized. Dehne et al. [11] showed that the area function has only a single local maximum inside the region where the set of Voronoi neighbors does not change, when the given points are in convex position. They gave a numerical algorithm for locating the optimal point based on Newton's approximation. Cheong et al. [9] proposed a near-linear time algorithm for the same problem that locates the new optimal point approximately, when the points in $S$ are in general position.

Our framework considers similar area maximization problems in a different scenario. In Section 2 we prove some basic results, which are used later in our analysis. In Section 3 we solve a restricted version of problem P1, where the points in $S$ are assumed to be placed equidistantly from the center $o$ of $\psi$. Under this assumption, Area $\left\{\bigcup_{i=1}^{n} V R\left(p_{i}, S \cup\{\psi\}\right)\right\}$ is maximized when the points in $S$ lie on the vertices of a regular $n$-gon with circumcenter at $o$. The optimum distance of the members in $S$ from $o$ and the optimum area increases with $n$, and at the limit approaches the radius and the area of the circle $\psi$, respectively. In Section 4, we study the second problem (Problem P2). We give an exact solution of the problem for $n=1$. Moreover, for $n \geq 1$, using the techniques of Cheong et al. [9], we give an $O\left(n / \varepsilon^{4}+n \log n\right)$ algorithm, which locates a point $x_{a}$ such that $\operatorname{Area}\left\{\operatorname{VR}\left(x_{a}, S \cup\left\{\psi, x_{a}\right\}\right)\right\} \geq$ $(1-\varepsilon)$ OPT Area, where OPT Area $=\sup _{x} \operatorname{Area}\{\operatorname{VR}(x, S \cup\{\psi, x\})\}$ and $\varepsilon>0$. Finally, in Section 5 we summarize our work and give some directions for future work. 


\section{Properties of a Voronoi Zone}

In this section, we prove some simple results regarding the Voronoi diagram $V(S \cup\{\psi\})$, where $\psi$ is a circle with radius $r$ and center $o$ and $S$ is a set of $n$ points placed inside $\psi$. We will use $\delta(a, b)$ to denote the Euclidean distance between the pair of points $a$ and $b$ and $\delta(a, \psi)$ to denote minimum distance of the point $a$ from the circumference of the circle $\psi$.

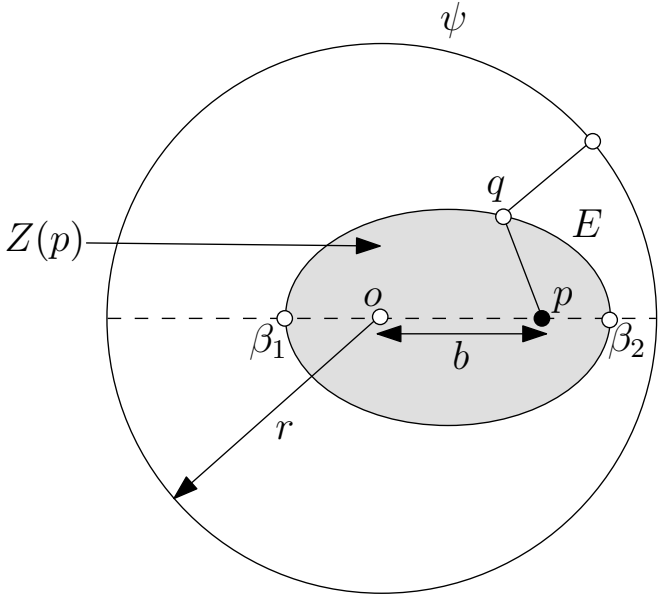

(a)

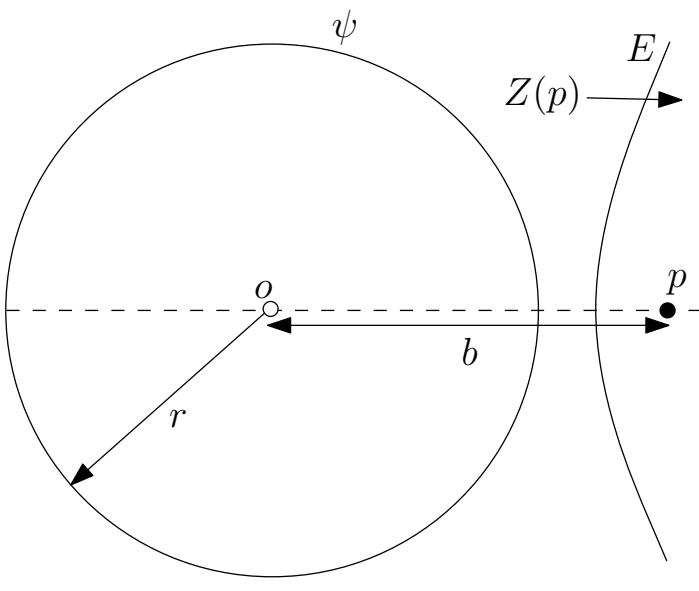

(b)

Fig. 2. The Voronoi region of $p$ : (a) point $p$ is inside $\psi$, and (b) point $p$ is outside $\psi$.

Let $p$ be a point placed inside the circle $\psi$. For any point $q$ on the boundary of $\operatorname{VR}(p,\{p, \psi\}), \delta(q, p)=\delta(q, \psi)=\delta\left(q, q^{\prime}\right)$, where $q^{\prime}$ is the point closest to $q$ on the circumference of $\psi$. Clearly, points $o, q, q^{\prime}$ must be collinear (Figure 2(a)). This implies, the locus $E$ of the point $q$ such $\delta(q, p)=\delta(q, \psi)$, satisfies $\delta(q, p)+\delta(q, o)=\delta\left(q, q^{\prime}\right)+\delta(q, o)=\delta\left(o, q^{\prime}\right)=r$. Thus, $E$ is an ellipse with foci the points $o$ and $p$, with $\left[\beta_{1}, \beta_{2}\right]$ as the major axis, where $\beta_{1}$ and $\beta_{2}$ are the points of intersection of $E$ with the diameter of $\psi$ passing through the point $p$. Therefore, the region enclosed by the ellipse $E$ is the Voronoi region of $p$ in $V(\{p, \psi\})$, where $p$ is a single point placed inside a circle $\psi$ with center $o$ and radius $r$ (Figure 2(a)).

Consider the rectangular coordinate system, with the origin at the point $o$ and the horizontal axis aligned along the diameter of the circle $\psi$ passing through the point $p$. Let the coordinates of the point $p$ be $(b, 0)$, where $b \geq 0$ is a non-negative constant. Then the equation of the locus $E$ can be easily shown to be:

$$
4 x^{2}\left(r^{2}-b^{2}\right)+4 r^{2} y^{2}-4 b x\left(r^{2}-b^{2}\right)-\left(r^{2}-b^{2}\right)^{2}=0 .
$$

The following lemma can now be proved from (1) using simple analytic geometry.

Lemma 1. The ellipse $E$ has the following properties:

(i) The length of the major axis of the ellipse $E$ is independent of the location of the fixed point $p$, and is equal to the radius $r$ of the circle $\psi$.

(ii) The length of the minor axis of $E$ is equal to $\sqrt{r^{2}-b^{2}}$, eccentricity $e=\frac{b}{r}$, and the foci are at $o=(0,0)$ and $p=(b, 0)$. 
In the limiting case, when the point $p$ coincides with the center $o$ (i.e. $b=0$ ) then $E$ becomes a circle of radius $r / 2$. In the other limiting case, when the point $p$ lies on the circle $\psi($ i.e. $b=r$ ) then $E$ is the radius of the circle through $p$, which can be interpreted as a degenerate ellipse with length of minor axis equal to zero. It is also interesting to note that if the point $p$ lies outside the circle $\psi$, the locus of the point $q$ satisfying $\delta(q, p)=\delta(q, \psi)$ is a hyperbola with foci at the points $o$ and $p$ (Figure 2(b)).

Next, we consider the situation where more than one point is placed inside the circle $\psi$. The following lemma states the nature of the elliptic zones when two points $p_{1}$ and $p_{2}$ are placed inside $\psi$. Let $E_{1}$ and $E_{2}$ be the loci of the point $q$ satisfying $\delta\left(q, p_{1}\right)=\delta(q, \psi)$ and $\delta\left(q, p_{2}\right)=\delta(q, \psi)$, respectively. From Lemma $1, E_{1}$ and $E_{2}$ are two ellipses with foci $o, p_{1}$ and $o, p_{2}$, respectively.

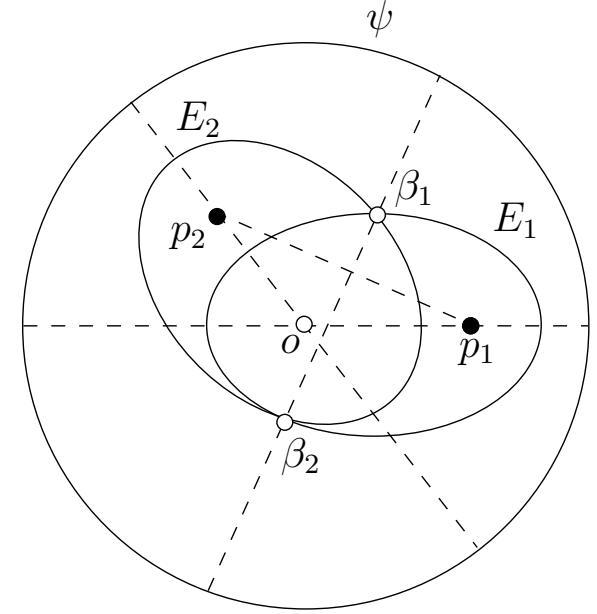

(a)

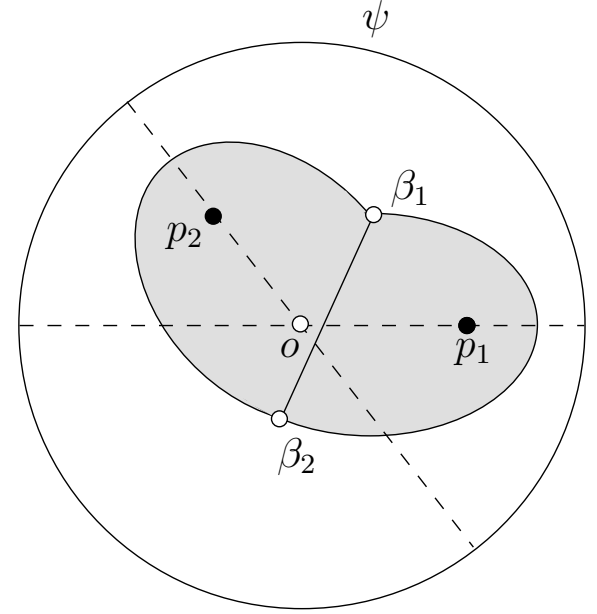

(b)

Fig. 3. (a) Proof of Lemma 2, and (b) Demonstration of $V\left(p_{1} \cup p_{2} \cup\{\psi\}\right)$.

Lemma 2. Let $p_{1}, p_{2}$ be two points placed inside the circle $\psi$, and $E_{1}, E_{2}$ be two ellipses with foci $o, p_{1}$ and $o, p_{2}$, respectively. The perpendicular bisector of the line joining $p_{1}$ and $p_{2}$ intersect the two ellipses $E_{1}$ and $E_{2}$ at a common chord.

Proof. Since $p_{1}$ and $p_{2}$ are two distinct points placed inside the circle $\psi$, and $r$ is the length of the major axes of both $E_{1}$ and $E_{2}$ (Lemma 1), none of the ellipses $E_{1}$ and $E_{2}$ properly contains the other. Thus, they must intersect in two distinct points, say $\beta_{1}$ and $\beta_{2}$ respectively (see Figure $3(\mathrm{a})$ ). Since $\beta_{1}$ lies on both $E_{1}$ and $E_{2}$, we have $\delta\left(\beta_{1}, p_{1}\right)=$ $\delta\left(\beta_{1}, \psi\right)=\delta\left(\beta_{1}, p_{2}\right)$. Thus, $\beta_{1}$ lies on the bisector of $p_{1}$ and $p_{2}$. Similarly, $\beta_{2}$ also lies on the bisector of $p_{1}$ and $p_{2}$. Since $E_{1}$ and $E_{2}$ are convex, $\left[\beta_{1}, \beta_{2}\right]$ is the common chord of the ellipses $E_{1}$ and $E_{2}$.

Observe that by the same argument as before, the points of intersection of the ellipses $E_{1}$ and $E_{2}$ must lie on the perpendicular bisector of the points $p_{1}$ and $p_{2}$. Now, since any line intersects an ellipse at most two points, $E_{1}$ and $E_{2}$ cannot intersect at more than two points.

The Voronoi diagram $V\left(\left\{p_{1}, p_{2}, \psi\right\}\right)$, is shown in Figure $3(\mathrm{~b})$. Since $\left[\beta_{1}, \beta_{2}\right]$ is the perpendicular bisector of the line segment $\left[p_{1}, p_{2}\right]$, the nearest service station for a user located 
in $Z\left(p_{1}\right)=V R\left(p_{1},\left\{p_{1}, p_{2}, \psi\right\}\right)$ is $p_{1}$. Similarly, the nearest service station for a user located in $Z\left(p_{2}\right)=V R\left(p_{2},\left\{p_{1}, p_{2}, \psi\right\}\right)$ is $p_{2}$.

\section{Problem P1}

In this section, our objective is to find the placement of the stationary service stations $S=\left\{p_{1}, p_{2}, \ldots, p_{n}\right\}$ inside the circle $\psi$ such that the total area service zone of the members in $S$ is maximized. This is equivalent to maximizing Area $\left\{\bigcup_{i=1}^{n} V R\left(p_{i}, S \cup\{\psi\}\right)\right\}$.

However, it is difficult to predict the behavior of the area of the union of the Voronoi regions of two points $p$ and $q$ placed arbitrarily inside $\psi$. This motivates us to consider a simple model where the members in $S$ are placed at same distances from the center $o$ of $\psi$. Under this assumption, the problem of maximizing Area $\left\{\bigcup_{i=1}^{n} V R\left(p_{i}, S \cup\{\psi\}\right)\right\}$ has the following two aspects:

(i) Assuming $\delta\left(o, p_{1}\right)=\delta\left(o, p_{2}\right)=\ldots=\delta\left(o, p_{n}\right)=b(>0)$, determine the optimal angles between the lines $\left[o, p_{i}\right]$ and $\left[o, p_{i+1}\right]$, for $i=1,2, \ldots, n$, such that Area $\left\{\bigcup_{i=1}^{n} V R\left(p_{i}, S \cup\right.\right.$ $\{\psi\})\}$ is maximized.

(ii) Determine the optimal length $\delta\left(o, p_{1}\right)\left(=\delta\left(o, p_{2}\right)=\ldots=\delta\left(o, p_{n}\right)\right)$ which maximizes Area $\left\{\bigcup_{i=1}^{n} V R\left(p_{i}, S \cup\{\psi\}\right)\right\}$.

We begin by showing that under the equidistant assumption Area $\left\{\bigcup_{i=1}^{n} V R\left(p_{i}, S \cup\{\psi\}\right)\right\}$ is maximized when all the $n$ points are placed on the regular $n$-gon with circumcenter at $o$.

Theorem 1. Let $S=\left\{p_{1}, p_{2}, \ldots, p_{n}\right\}$ be a set of stationary service stations placed inside a circle $\psi$ with center $o$ and radius $r$, such that $\delta\left(o, p_{i}\right)=b$ for all $i=1,2, \ldots, n$, where $b$ $(<r)$ is a given positive constant. Then Area $\left\{\bigcup_{i=1}^{n} V R\left(p_{i}, S \cup\{\psi\}\right)\right\}$ is maximized when the members in $S$ lie on the vertices of a regular n-gon with circumcenter at $o$.

Proof. Let $p$ be any point inside $\psi$ such that $\delta(o, p)=b$, and $E$ be any ellipse with foci at $o$ and $p$. The problem of maximizing Area $\left\{\bigcup_{i=1}^{n} V R\left(p_{i}, S \cup\{\psi\}\right)\right\}$ is equivalent to choosing angles $\phi_{1}, \ldots, \phi_{n}$ such that Area $\left\{\bigcup_{i=1}^{n} E_{\phi_{i}}\right\}$ is maximized, where $E_{\phi}$ denotes a copy of $E$ rotated by an angle $\phi$ about the center $o$ in the clockwise direction.

For $0 \leq u \leq r$, denote by $C_{u}$ the circumference of the circle with center at the point $o$ and radius $u$. Observe that Area $\left\{\bigcup_{i=1}^{n} E_{\phi_{i}}\right\}=\int_{0}^{r} \lambda(v) d v$, where $\lambda(v)$ is the length of the curve $\bigcup_{i=1}^{n} E_{\phi_{i}} \cap C_{v}$. It is easy to verify from Equation (1) that for every $v \geq 0, C_{v}$ intersects the ellipse $E$ in at most one circular arc. Observe that if $n$ arcs of equal length are to be chosen on some circle, then a regular distribution of these arcs about the circle maximizes the length of their union. This is because, under a regular distribution of the arcs, the length of the curve $\bigcup_{i=1}^{n} E_{\phi_{i}} \cap C_{v}$ is either the length of the circumference of $C_{v}$, or the sum of the lengths of $E_{\phi_{i}} \cap C_{v}$, for every $0 \leq v \leq r$. This implies that for each $v \geq 0$ the function $\lambda(v)$ is maximized with a regular distribution of the $n$ angles $\phi_{1}, \phi_{2}, \ldots, \phi_{n}$.

Therefore, Area $\left\{\bigcup_{i=1}^{n} E_{\phi_{i}}\right\}$ is maximized when $\phi_{i}=2 i \pi / n$, for $i=1,2, \ldots, n$, and the result follows.

Observe that for each $p_{i} \in S, V R\left(p_{i}, S \cup\{\psi\}\right)$ can be divided into two elliptical subsectors by the line segment $\left[o, p_{i}\right]$. Let $\theta_{2 i-1}$ and $\theta_{2 i}$ be the angles subtended by these subsectors at the center $o$ of $\psi$ as shown in Figure 4(a). Observe that $\sum_{i=1}^{2 n} \theta_{i}=2 \pi$, and Theorem 1 implies that Area $\left\{\bigcup_{i=1}^{n} \operatorname{VR}\left(p_{i}, S \cup\{\psi\}\right)\right\}$ is maximized if $\theta_{1}=\theta_{2}=\ldots=\theta_{2 n}=\pi / n$. In fact, we can use this observation to obtain a simple analytic proof of Theorem 1 , as 


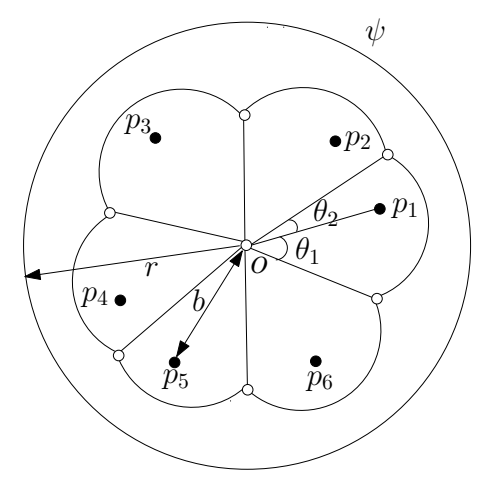

(a)

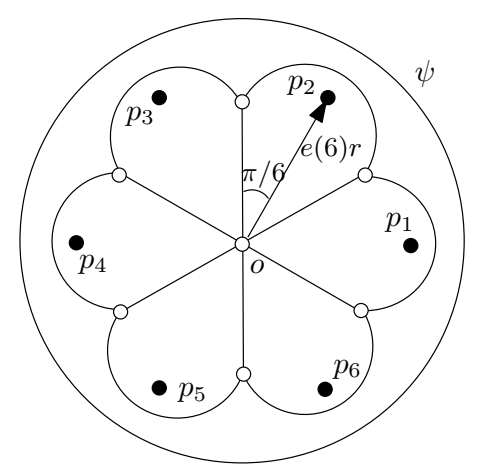

(b)

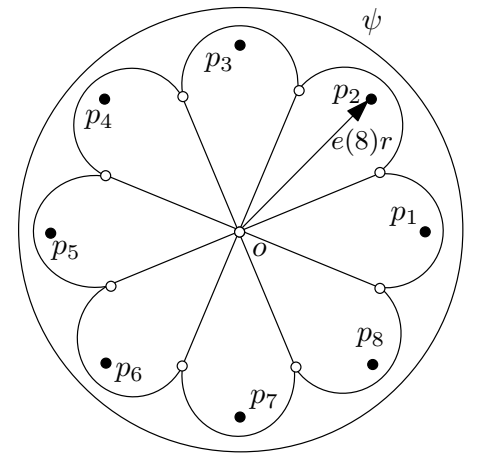

(c)

Fig. 4. Problem P1: (a) $V(S \cup\{\psi\})$ when the points in $S$ are equidistant from $o$ but not equiangular, (b) $V(S \cup\{\psi\})$, with $|S|=6$, when Area $\left\{\bigcup_{i=1}^{6} V R\left(p_{i}, S \cup\{\psi\}\right)\right\}$ is maximized, and (c) $V(S \cup\{\psi\})$, with $|S|=8$, when Area $\left\{\bigcup_{i=1}^{8} V R\left(p_{i}, S \cup\{\psi\}\right)\right\}$ is maximized.

described below.

Remark 1: For an ellipse with origin at one of its foci, eccentricity $e$ and semi-latus rectum $l$, its polar equation is $\rho=\frac{l}{1-e \cos \theta}[14]$. By Lemma 1 , we have $e=\frac{b}{r}$ and $l=\frac{r^{2}-b^{2}}{2 r}$. Define a function $\mathcal{F}:[0, \pi] \rightarrow \mathbb{R}$ as follows:

$$
\mathcal{F}(x)=\frac{1}{2} \int_{0}^{x} \rho^{2} d \theta=\frac{r^{2}}{8}\left[\frac{e\left(1-e^{2}\right) \sin x}{1-e \cos x}+2 \sqrt{1-e^{2}} \tan ^{-1}\left(\sqrt{\frac{1+e}{1-e}} \tan \frac{x}{2}\right)\right] .
$$

Then Area $\left\{\bigcup_{i=1}^{n} \operatorname{VR}\left(p_{i}, S \cup\{\psi\}\right)\right\}=\mathcal{A}(\boldsymbol{\Theta})=\sum_{i=1}^{2 n} \mathcal{F}\left(\theta_{i}\right)=\sum_{i=1}^{2 n-1} \mathcal{F}\left(\theta_{i}\right)+\mathcal{F}(\eta)$, where $\eta=2 \pi-\sum_{i=1}^{2 n-1} \theta_{i}$ and $\boldsymbol{\Theta}=\left(\theta_{1}, \theta_{2}, \ldots, \theta_{2 n-1}\right)$. To maximize $\mathcal{A}(\boldsymbol{\Theta})$ we need to solve the system of equations: $\frac{\partial \mathcal{A}}{\partial \theta_{i}}=\frac{\partial}{\partial \theta_{i}} \mathcal{F}\left(\theta_{i}\right)-\frac{\partial}{\partial \eta} \mathcal{F}(\eta)=0$, for $i=1,2, \ldots, 2 n-1$. Observe that if $\boldsymbol{\Theta}=\boldsymbol{\Theta}_{0}=(\pi / n, \pi / n, \ldots, \pi / n)$, then $\eta=\pi / n$ and $\frac{\partial \mathcal{A}}{\partial \theta_{i}}=0$ for all $i=1,2, \ldots, 2 n-1$. This and the concavity of the function proved below implies that $\Theta_{0}$ uniquely maximizes the function $\mathcal{A}$.

Now, we show that function $\mathcal{A}(\boldsymbol{\Theta})$ is concave, which immediately implies that the Area $\left\{\bigcup_{i=1}^{n} V R\left(p_{i}, S \cup\{\psi\}\right)\right\}$ has a unique maximum at $\theta_{1}=\theta_{2}=\ldots=\theta_{2 n}=\pi / n$.

Theorem 2. The function $\mathcal{A}(\boldsymbol{\Theta})$ is concave on the set $C:=[0, \pi]^{2 n-1} \subset \mathbb{R}^{2 n-1}$.

Proof. Observe that $\mathcal{A}(\boldsymbol{\Theta})=\sum_{i=1}^{2 n-1} \mathcal{F}\left(\theta_{i}\right)+\mathcal{F}(\eta)$, where $\eta=2 \pi-\sum_{i=1}^{2 n-1} \theta_{i}$. Now, since the sum of concave functions is also a concave function, to show that $\mathcal{A}(\boldsymbol{\Theta})$ is concave on $C$ it suffices to prove that the function $\mathcal{F}(x)$ is concave on $[0, \pi]$. Since $\mathcal{F}(x)$ is twice continuously differentiable on $[0, \pi]$, it follows from (2) by taking derivatives that

$$
\mathcal{H}(x)=\frac{d^{2}}{d^{2} x} \mathcal{F}(x)=-\frac{r^{2}}{4}\left[\frac{e\left(1-e^{2}\right)^{2} \sin x}{(1-e \cos x)^{3}}\right] .
$$

Now, since as $e \in[0,1]$ and $\sin x \geq 0$ for $x \in[0, \pi]$, it follows that $\mathcal{H}(x) \leq 0$, for $x \in[0, \pi]$. This establishes the concavity of the function $\mathcal{F}(x)$ on $[0, \pi]$ (see Figure $5(\mathrm{a})$ ), Therefore, $\mathcal{A}(\boldsymbol{\Theta})=\operatorname{Area}\left\{\bigcup_{i=1}^{n} V R\left(p_{i}, S \cup\{\psi\}\right)\right\}$ is concave on $C$. 
We now present the procedure for computing the optimal length $b\left(=\delta\left(o, p_{i}\right)\right)$ for all $i=1,2, \ldots, n$, where $n$ is given. Theorem 1 says that when the maximum is attained Area $\left\{\operatorname{VR}\left(p_{i}, S \cup\{\psi\}\right)\right\}$ is the same for all $i=1,2, \ldots, n$. Hence, using the polar equation of the ellipse [14], we may write $\operatorname{Area}\left\{\bigcup_{i=1}^{n} V R\left(p_{i}, S \cup\{\psi\}\right)\right\}=n \int_{0}^{\pi / n} \rho^{2} d \theta$ as a function $\mathcal{K}(e, n)$ of the eccentricity $e$ as follows:

$$
\mathcal{K}(e, n)=\frac{n r^{2}}{4}\left[\frac{e\left(1-e^{2}\right) \sin \frac{\pi}{n}}{1-e \cos \frac{\pi}{n}}+2 \sqrt{1-e^{2}} \tan ^{-1}\left(\sqrt{\frac{1+e}{1-e}} \tan \frac{\pi}{2 n}\right)\right] .
$$

Finding the optimum value of $b$ is equivalent to maximizing $\mathcal{K}(e, n)$ with respect to $e$, or equivalently solving the equation $\frac{d}{d e} \mathcal{K}(e, n)=0$. By checking the non-positivity of the second derivative, it can be easily verified that the function $\mathcal{K}(e, n)$ is concave in $e$, which implies $\mathcal{K}(e, n)$ has an unique global maximum.

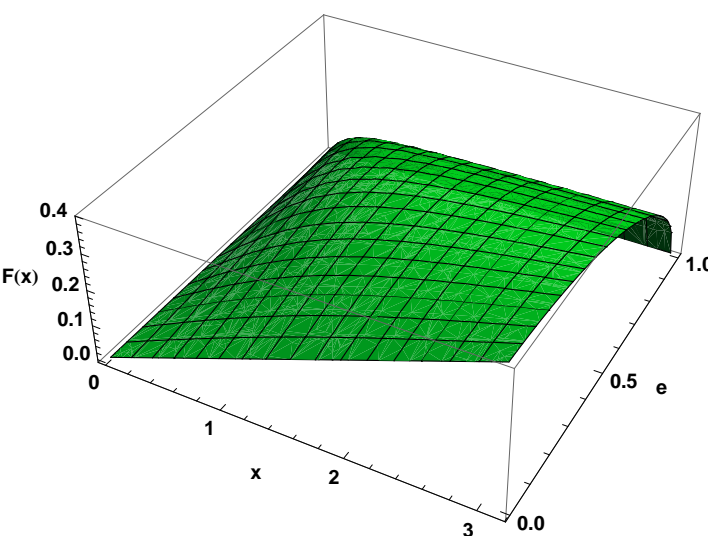

(a)

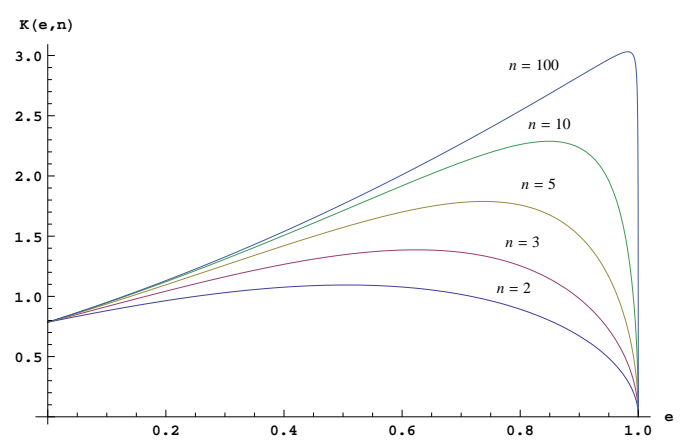

(b)

Fig. 5. Experimental results: (a) $3 \mathrm{D}$ plot of $\mathcal{F}(x)$ as $e$ varies from 0 to 1 , (b) Plot of $\mathcal{K}(e, n)$ for different values of $n$ and $r=1$

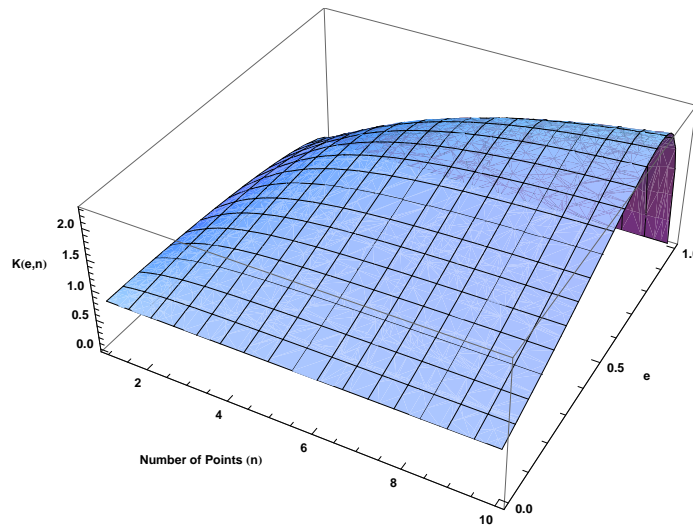

(a)

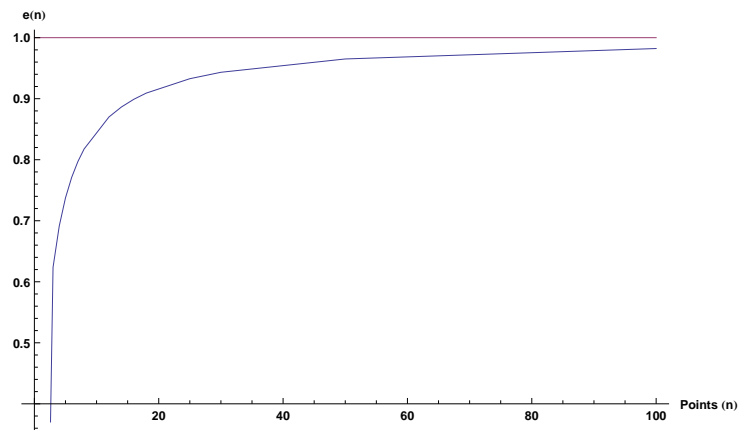

(b)

Fig. 6. Experimental results: (a) $3 \mathrm{D}$ plot of $\mathcal{K}(e, n)$ for a unit circle, and (b) asymptotic behavior of $e(n)$.

The optimum values of $e$ for different values of $n$, to be denoted by $e(n)$, are obtained by solving the equation $\frac{d}{d e} \mathcal{K}(e, n)=0$ numerically using Mathematica 4.0. Table 1 lists the 
values $e(n)$ and the corresponding values of $\mathcal{K}(e(n), n))=\operatorname{Area}\left\{\bigcup_{i=1}^{n} V R\left(p_{i}, S \cup\{\psi\}\right)\right\}$ for different values of $n$. Figure $5(\mathrm{~b})$ shows a plot of $\mathcal{K}(e, n)$ for different values of $n$, where $\psi$ is a unit radius circle. Figure 6(a) shows another $3 \mathrm{D}$ plot $\mathcal{K}(e, n)$ fixing $r=1$.

Table 1. The optimum ratio $e$ and the corresponding area for various $n$

\begin{tabular}{|c|c|c|}
\hline$n$ & $e=\frac{b}{r}$ & Area $\left\{\bigcup_{i=1}^{n} V R\left(p_{i}, S \cup\{\psi\}\right)\right\}$ \\
\hline 1 & 0.000000 & $0.78539 r^{2}$ \\
\hline 2 & 0.505879 & $1.09446 r^{2}$ \\
\hline 3 & 0.623606 & $1.38652 r^{2}$ \\
\hline 4 & 0.691382 & $1.61193 r^{2}$ \\
\hline 5 & 0.737416 & $1.78829 r^{2}$ \\
\hline 6 & 0.771153 & $1.92921 r^{2}$ \\
\hline 7 & 0.797069 & $2.04409 r^{2}$ \\
\hline 8 & 0.817647 & $2.13939 r^{2}$ \\
\hline 50 & 0.965112 & $2.92771 r^{2}$ \\
\hline 100 & 0.982216 & $3.03124 r^{2}$ \\
\hline 500 & 0.996387 & $3.11895 r^{2}$ \\
\hline 1000 & 0.998190 & $3.13030 r^{2}$ \\
\hline
\end{tabular}

Table 1 shows that as $n$ increases the optimum ratio $e(n)$ increases (see Figure 4(a) and Figure 4(b)) and at the limit reaches 1 (Figure 6(b)). Table 1 also demonstrates that the optimum area of the combined service zone of all the members in $S$ increases to $\pi r^{2}$ asymptotically.

We give formal proofs of these experimental observations in the following theorem:

Theorem 3. If $e(n)$ denotes the value of $e$ at which the function $\mathcal{K}(e, n)$ attains its maximum, then (a) $e\left(n_{1}\right)<e\left(n_{2}\right)$, for positive integers $n_{1}<n_{2}$, (b) $\lim _{n \rightarrow \infty} e(n)=1$, (c) $\mathcal{K}\left(e\left(n_{1}\right), n_{1}\right)<\mathcal{K}\left(e\left(n_{2}\right), n_{2}\right)$ for positive integers $n_{1}<n_{2}$, and $(d) \lim _{n \rightarrow \infty} \mathcal{K}(e, n)=\pi r^{2}$.

Proof. Let $\mathcal{K}^{\prime}(e, n)=\frac{d}{d e} \mathcal{K}(e, n)$, where $\mathcal{K}(e, n)=n \int_{0}^{\pi / n} \rho^{2} d \theta=\frac{n r^{2}}{4} \int_{0}^{\pi / n} \frac{\left(1-e^{2}\right)^{2}}{(1-e \cos \theta)^{2}} d \theta$. Interchanging the order of the derivative and the integration [Theorem 7.40, Apostol [3]], we get

$$
\mathcal{K}^{\prime}(e, n)=n \int_{0}^{\pi / n} \frac{d}{d e} \rho^{2} d \theta=\frac{n r^{2}\left(1-e^{2}\right)}{2} \int_{0}^{\pi / n} \frac{\left(1+e^{2}\right) \cos \theta-2 e}{(1-e \cos \theta)^{3}} d \theta .
$$

(a) Let $\mathcal{A}(e, \theta)=\frac{\left(1+e^{2}\right) \cos \theta-2 e}{(1-e \cos \theta)^{3}}$. As $0 \leq e<1, \mathcal{K}(e, n)=0$ if and only if $\int_{0}^{\pi / n} \mathcal{A}(e, \theta) d \theta=0$. Consider two positive integers $1<n_{1}<n_{2}$. Since $\mathcal{K}\left(e, n_{2}\right)$ is concave for $e \in[0,1]$ it follows that $\mathcal{K}^{\prime}\left(e, n_{2}\right)$ is non-increasing for $e \in[0,1]$. Observe that $\mathcal{A}\left(e\left(n_{1}\right), \theta\right)=0$ if $\cos \theta=2 e\left(n_{1}\right) /\left(1+e\left(n_{1}\right)^{2}\right)$. This equation has an unique solution for $\theta \in[0, \pi / n]$, which implies that the equation $\mathcal{A}(e, \theta)=0$ also has an unique solution for $\theta \in[0, \pi / n]$. Now, since $\mathcal{A}\left(e\left(n_{1}\right), 0\right)=1 /\left(1-e\left(n_{1}\right)\right)>0, \int_{0}^{\pi / n_{1}} \mathcal{A}\left(e\left(n_{1}\right), \theta\right) d \theta=0$, and $\left[0, \pi / n_{2}\right] \subset$ $\left[0, \pi / n_{1}\right]$, it follows that $\int_{0}^{\pi / n_{2}} \mathcal{A}\left(e\left(n_{1}\right), \theta\right) d \theta>0$. Therefore, $\mathcal{K}^{\prime}\left(e\left(n_{1}\right), n_{2}\right)>0$. Hence, $\mathcal{K}^{\prime}\left(e\left(n_{2}\right), n_{2}\right)=0<\mathcal{K}^{\prime}\left(e\left(n_{1}\right), n_{2}\right)$ which implies that $e\left(n_{1}\right)<e\left(n_{2}\right)$, since $\mathcal{K}^{\prime}\left(e, n_{2}\right)$ is non-increasing in $e$.

(b) As $e(n)$ increases with $n$ and each $e(n)<1$ for all $n \geq 1$, it follows that $\lim _{n \rightarrow \infty} e(n)$ exists and is at most 1 . Observe that there exists a positive integer $N(e)$ such that $\cos \theta>2 e /\left(1-e^{2}\right)$ whenever $\theta \in[0, \pi / n]$ for all $n \geq N(e)$. This implies that whenever 
$n \geq N(e)$, we have $\left(1-e^{2}\right) \cos \theta-2 e>0$ for $\theta \in[0, \pi / n]$. Therefore, for all $n \geq N(e)$, $\mathcal{K}^{\prime}(e, n)>0$. This means that given any $\varepsilon>0$, we have a positive integer $N(\varepsilon)$ such that $\mathcal{K}^{\prime}(1-\varepsilon, n)>0$, whenever $n \geq N(\varepsilon)$. Now, since $\mathcal{K}^{\prime}(e, n)$ is non-increasing in $e$ and $\mathcal{K}^{\prime}(e(n), n)=0$, we have $e(n)>1-\varepsilon$, whenever $n \geq N(\varepsilon)$. This proves that $\lim _{n \rightarrow \infty} e(n)=1$.

(c) Observe that $\mathcal{K}(e(n), n)=\operatorname{Area}\left(\bigcup_{i=1}^{n} V R\left(p_{i}, S \cup\{\psi\}\right)\right)$, where the points $p_{i} \in S$ are placed on the vertices of a regular polygon at a distance $e(n)$ from the center $o$ of the circle $\psi$. Let $q \notin S$ be another point such that $\delta(o, q)=e(n)$. Then $\bigcup_{i=1}^{n} V R\left(p_{i}, S \cup\right.$ $\{\psi\}) \subset\left(\bigcup_{i=1}^{n} V R\left(p_{i}, S \cup\{q, \psi\}\right)\right) \cup V R(q, S \cup\{q, \psi\})$, and we have

$$
\mathcal{K}(e(n), n)<\operatorname{Area}\left(\left(\bigcup_{i=1}^{n} \operatorname{VR}\left(p_{i}, S \cup\{q, \psi\}\right)\right) \cup V R(q, S \cup\{q, \psi\})\right) \leq \mathcal{K}(e(n+1), n+1) .
$$

(d) Using the facts that for some constant $a, \lim _{n \rightarrow \infty} n \sin (a / n)=a, \lim _{n \rightarrow \infty} n \tan ^{-1}(a / n)=$ $a$, and $\lim _{n \rightarrow \infty} n \tan (a / n)=a$ we get, for every fixed $e \in[0,1)$

$$
\lim _{n \rightarrow \infty} \mathcal{K}(e, n)=\left(r^{2} / 4\right)[\pi e(1+e)+\pi(1+e)]=\frac{\pi r^{2}(1+e)^{2}}{4}=\ell(e) .
$$

Now, let $\varepsilon>0$ be given and define $\delta=\varepsilon /\left(\pi r^{2}+1\right)$. Since $\lim _{n \rightarrow \infty} e(n)=1$, we can find a positive integer $N(\varepsilon)$ such that $e(n)>1-\delta$ and $\mathcal{K}(1-\delta, n)>\ell(1-\delta)-\delta$, whenever $n \geq N(\varepsilon)$. It now follows from (c) that $\mathcal{K}(e(n), n) \geq \mathcal{K}(1-\delta, n)>\ell(1-\delta)-\delta=$ $\left(\pi r^{2} / 4\right)(2-\delta)^{2}-\delta>\pi r^{2}-\left(\pi r^{2} \delta+\delta\right)+\pi \delta^{2} / 4>\pi r^{2}-\left(\pi r^{2} \delta+\delta\right)=\pi r^{2}-\varepsilon$, whenever $n \geq N(\varepsilon)$. This proves $\lim _{n \rightarrow \infty} \mathcal{K}(e, n)=\pi r^{2}$.

\section{Problem P2}

In this section, we address another variation of the facility location problem involving both mobile and stationary service stations. Given a circle $\psi$ and a set of $n$ points $S=$ $\left\{p_{1}, p_{2}, \ldots, p_{n}\right\}$ placed inside $\psi$, the task is to locate a new point $q$ inside $\psi$ such that its Voronoi region Area $\{\operatorname{VR}(q, S \cup\{q, \psi\})\}$ is maximized. We give an exact solution of the problem when there is only one existing stationary service station, that is, when $n=1$. For any arbitrary $n \geq 1$, we give an approximation algorithm which locates a point $x_{a}$ such that Area $\left\{\operatorname{VR}\left(x_{a}, S \cup\left\{\psi, x_{a}\right\}\right)\right\} \geq(1-\varepsilon)$ OPT Area, where OPTArea $=\sup _{x}$ Area $\{V R(x, S \cup$ $\{\psi, x\})\}$.

\subsection{Exact Solution for $n=1$}

Let us choose a coordinate system where the point $o$ is the origin, and the line joining $o$ and the point $p$ is the $x$-axis. Let $p=(-a, 0)$, where $a(<r)$ is a given positive constant. For a pair of points $q=(x, y)$ and $q^{\prime}=(-x, y)$ with $x>0$, we have $\operatorname{Area}\left(\operatorname{VR}\left(q^{\prime},\left\{p, q^{\prime}, \psi\right\}\right) \leq \operatorname{Area}(\operatorname{VR}(q,\{p, q, \psi\})\right.$. So, it is sufficient to consider only the positive values of $x$ for determining the optimal location of the point $q$. We can view this as a two variate optimization problem, where the variables are: $\eta=$ the angle between the line segments $[o, q]$ and $[o, p]$, and $b=\delta(o, q)$.

We begin with the following observation:

Observation 1 Let $E$ be an ellipse with foci at the points $f_{1}, f_{2}$ and center point o. Let $\beta_{1}$, $\beta_{2}$ be two distinct points on the line segment $\left[o, f_{1}\right]$ such that $\delta\left(f_{1}, \beta_{1}\right)<\delta\left(f_{1}, \beta_{2}\right)$. Suppose 


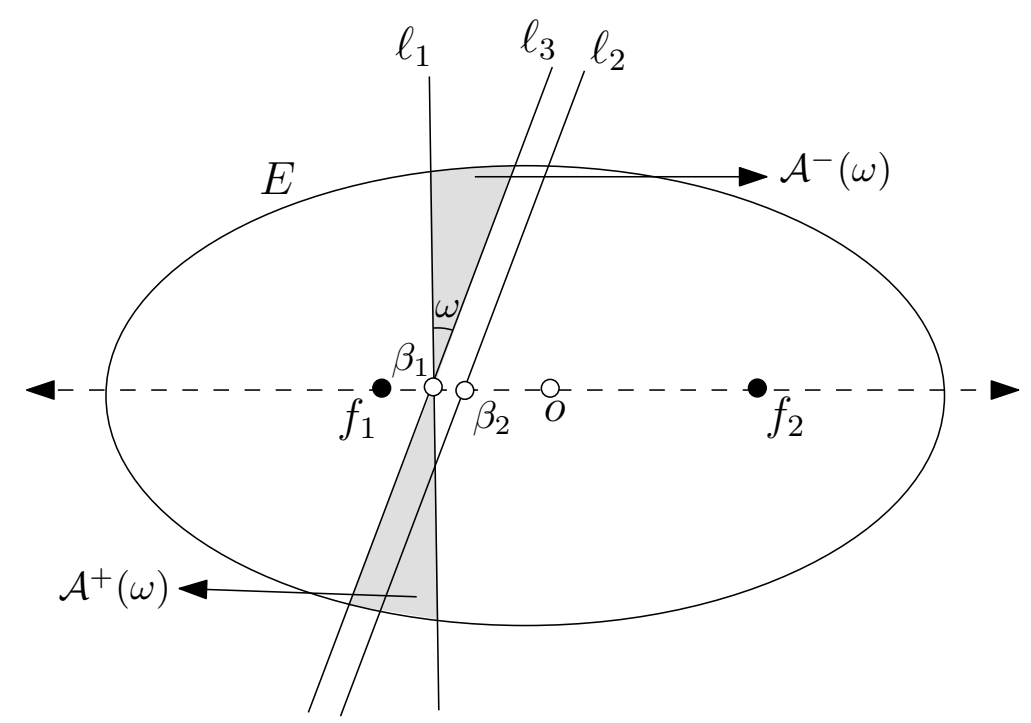

Fig. 7. Proof of Observation 1.

$\ell_{1}$ and $\ell_{2}$ are lines through $\beta_{1}$ and $\beta_{2}$ respectively, such that $\ell_{1}$ is perpendicular to $\left[o, f_{1}\right]$ and angle between $\ell_{2}$ and $\left[o, f_{1}\right]$ is acute. If $\mathcal{A}_{1}$ (resp. $\left.\mathcal{A}_{2}\right)$ denotes the area of the elliptic sector containing both $o$ and $f_{2}$ and bounded by the line $\ell_{1}$ (resp. $\ell_{2}$ ), then $\mathcal{A}_{1}>\mathcal{A}_{2}$.

Proof. Let $\ell_{3}$ be the line through $\beta_{1}$ which is parallel to $\ell_{2}$ and $\omega$ be the acute angle between $\ell_{1}$ and $\ell_{3}$. The polar equation of the ellipse $E$ with center at $o$ is $\rho=\frac{\lambda}{1-e \cos \theta}$, where $\lambda$ is a constant independent of $\theta$. Let $\mathcal{A}_{3}$ denote the area of the elliptic sector containing both $o$ and $f_{2}$ and bounded by the line $\ell_{3}$. Also, by $\mathcal{A}^{-}(\omega)$ and $\mathcal{A}^{+}(\omega)$ we denote the areas of the elliptic sectors enclosed by the lines $\ell_{1}$ and $\ell_{3}$ above and below the major axis of $E$, respectively (see Figure 7 ).

For $0<\alpha<\pi / 2$, define the function $\mathcal{A}^{\alpha}:[0, \pi / 2] \rightarrow \mathbb{R}$ as $\mathcal{A}^{\alpha}(x)=\frac{1}{2} \int_{x}^{x+\alpha} \rho^{2} d \theta$. By plugging in the polar equation of $E$ and taking the derivative of $\mathcal{A}^{\alpha}$ with respect to $x$, it follows that $\mathcal{A}^{\alpha}$ is non-increasing function of $x$ on $[0, \pi / 2]$. This implies that $\mathcal{A}^{+}(\omega)=$ $\mathcal{A}^{\omega}(\pi / 2) \leq \mathcal{A}^{\omega}(\pi / 2-\omega)=\mathcal{A}^{-}(\omega)$, since $0<\omega<\pi / 2$. Therefore, $\mathcal{A}_{2}<\mathcal{A}_{3}=\mathcal{A}_{1}+\mathcal{A}^{+}(\omega)-$ $\mathcal{A}^{-}(\omega) \leq \mathcal{A}_{1}$.

Lemma 3. Let $\psi$ be a circle with center $o$ and radius $r$, and $p=(-a, 0)$, be a stationary service station inside $\psi$. If $q$ is another service station placed inside $\psi$ such that $\delta(o, q)=b$, $(0<b<r)$, then Area $\{\operatorname{VR}(q,\{p, q, \psi\})\}$ is maximized when $\eta=\angle p o q=\pi$.

Proof. Let $\phi$ be a circle with center at $o$ and radius $b$ that intersects the positive side of the $x$ axis at point $q=(b, 0)$. We need to show that Area $\left\{V R\left(q^{\prime},\left\{p, q^{\prime}, \psi\right\}\right)\right\} \leq \operatorname{Area}\{V R(q,\{p, q, \psi\})\}$, where $q^{\prime} \neq q$ is any other point on boundary of $\phi$. If $\angle q^{\prime} o q=\theta$, the coordinates of the point $q^{\prime}$ are $(b \cos \theta, b \sin \theta)$. The ellipses $E_{1}, E_{2}$ and $E_{2}^{\prime}$ with one of the foci at $o$ and the other at $p, q$ and $q^{\prime}$ respectively, are shown in Figure 8(a).

Throughout the proof of this lemma, we shall refer to Figure 8(a). The equation of the perpendicular bisector of line segment $\left[p, q^{\prime}\right]$ (denoted by $u v$ in Figure $8(\mathrm{a})$ ) is $y-\frac{b \sin \theta}{2}=$ $-\frac{b \cos \theta+a}{b \sin \theta}\left(x-\frac{b \cos \theta-a}{2}\right)$. Let the line $o q^{\prime}$ intersect the line $u v$ at a point $\beta^{\prime}$. Note that, $q^{\prime} \beta^{\prime} \gamma$, where $\gamma$ is the point of intersection of the lines $p q^{\prime}$ and $u v$, is a right angled triangle, with right angle at the point $\gamma$. Therefore, $\angle \gamma \beta^{\prime} q^{\prime}$ is acute. 


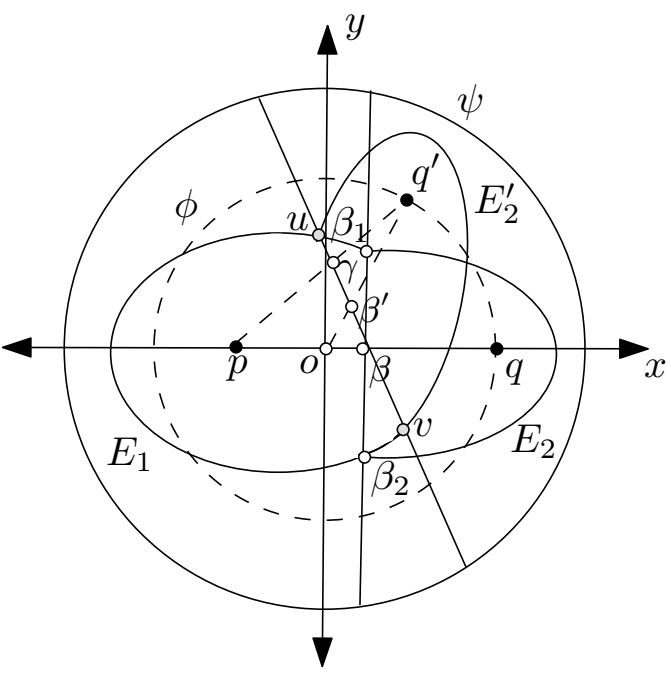

(a)

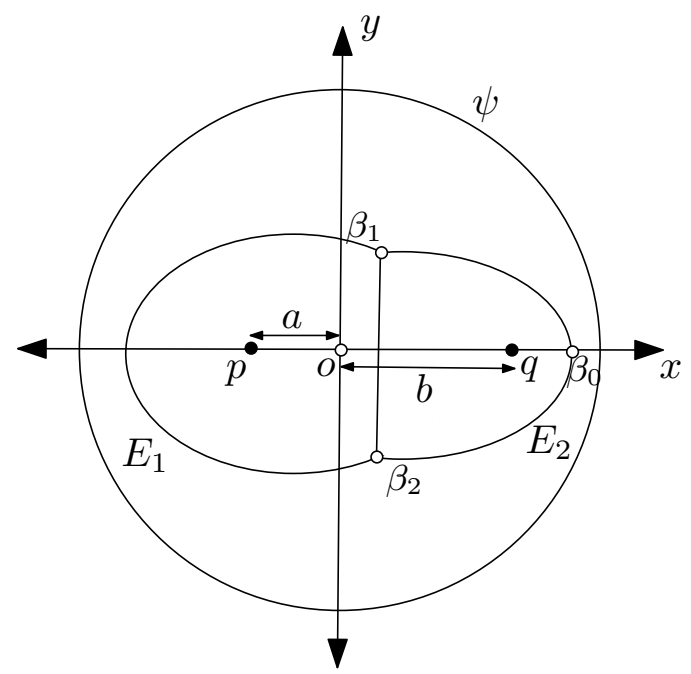

(b)

Fig. 8. (a) Proof of Lemma 3, and (b) Calculation of Area $\{\operatorname{VR}(q,\{p, q, \psi\})\}$

We first assume that $b \geq a$. Let $\left[\beta_{1}, \beta_{2}\right]$ be the perpendicular bisector of the line segment $[p, q]$ that intersects $o q$ at the point $\beta=\left(\frac{b-a}{2}, 0\right)$ and $\delta(o, \beta)=$ the length of the line segment $[o, \beta]=\left|\frac{b-a}{2}\right|$. Now, the length of the line segment $\left[o, \beta^{\prime}\right]=\frac{b^{2}-a^{2}}{2(b+a \cos \theta)} \geq \frac{b-a}{2}$. Thus, we have $\delta\left(o, \beta^{\prime}\right) \geq \delta(o, \beta)$ (Figure 8(a)).

$\operatorname{Area}\left(\operatorname{VR}(q,\{p, q, \psi\})\right.$ is the region bounded by the ellipse $E_{2}$ and the chord $\left[\beta_{1}, \beta_{2}\right]$, and $\operatorname{Area}\left\{V R\left(q^{\prime},\left\{p, q^{\prime}, \psi\right\}\right)\right\}=$ the region bounded by the ellipse $E_{2}^{\prime}$ and the chord $[u, v]$. Since the ellipse $E_{2}^{\prime}$ is just a rotation of the ellipse $E_{2}$, Observation 1 now implies that Area $\left\{\operatorname{VR}\left(q^{\prime},\left\{p, q^{\prime}, \psi\right\}\right)\right\} \leq \operatorname{Area}\{V R(q,\{p, q, \psi\})\}$. This completes the proof of the result when $b \geq a$.

The proof of the result for the case $b<a$ is similar.

Lemma 3 says that if $\delta(o, q)=b$ is fixed, Area $\{\operatorname{VR}(q,\{p, q, \psi\})\}$ attains a maximum when $\angle p o q=\pi$. We now compute the value of $b$ that maximizes $\operatorname{Area}(\operatorname{VR}(q,\{p \cup q, \psi\}))$. Let $E_{1}$ and $E_{2}$ respectively denote the elliptical sectors formed by the points $p$ and $q$ in the Voronoi diagram $V(\{p, q, \psi\})$ (Figure 8(b)). Since the common chord of the ellipses $E_{1}$ and $E_{2}$ is the perpendicular bisector of the line segment $[p, q]$ (Lemma 2), the $x$-coordinates of $\beta_{0}$ and $\beta_{1}$ are $\frac{b+r}{2}$ and $\frac{b-a}{2}$, respectively (see Figure $8(\mathrm{~b})$ ). Integrating equation of the ellipse $E_{2}: 4 x^{2}\left(r^{2}-b^{2}\right)+4 r^{2} y^{2}-4 b x\left(r^{2}-b^{2}\right)-\left(r^{2}-b^{2}\right)^{2}=0$, from $\beta_{0}$ to $\beta_{1}$, we have

$$
\operatorname{Area}(\operatorname{VR}(q,\{p, q, \psi\}))=\frac{1}{r} \int_{\frac{b-a}{2}}^{\frac{r+b}{2}} \sqrt{\left(r^{2}-b^{2}\right)^{2}+4 b x\left(r^{2}-b^{2}\right)-4 x^{2}\left(r^{2}-b^{2}\right)} d x .
$$

Simplifying and substituting $2 x-b=y$, the above integral reduces to

$$
\operatorname{Area}(\operatorname{VR}(q,\{p, q, \psi\}))=\frac{\sqrt{r^{2}-b^{2}}}{r}\left(\int_{-a}^{r} \sqrt{r^{2}-y^{2}} d y\right)=\lambda \frac{\sqrt{r^{2}-b^{2}}}{r},
$$

where $\lambda=\int_{-a}^{r} \sqrt{r^{2}-y^{2}} d y$ is a constant independent of $b$. Hence, the area of the Voronoi region of $q$ attains a maximum when $b=0$. This leads to the following theorem: 
Theorem 4. Let $p$ be a stationary service station placed inside a circle $\psi$ of radius $r$ and center at $o$, and $\delta(o, p)=a$, where $a(<r)$ is a given positive constant. While placing another service station $q$ inside $\psi$, Area $\{V R(q,\{p, q, \psi\})\}$ is maximized if $q$ is placed at the center o of $\psi$.

Note that even if $p$ is placed at the center $o$ of $\psi$, the optimum position of $q$ will be at $o$ itself, because our objective is to maximize the Voronoi zone of $q$ alone. When both the points $p$ and $q$ coincide with $o$, it is natural to assume that one half of the Voronoi region is served by $p$ while the other half is served by $q$. Therefore, when $p$ is placed at $o, \operatorname{Area}\{\operatorname{VR}(q,\{p, q, \psi\})\}$ is maximized when $q$ coincides with $p$ at $o$ and $\operatorname{Area}\{\operatorname{VR}(q,\{p, q, \psi\})\}=\frac{\pi r^{2}}{8}$.

\subsection{Approximate Solution of Problem P2}

Let $S=\left\{p_{1}, p_{2}, \ldots, p_{n}\right\}$ be a set of $n$ points placed inside the unit circle $\psi$ with center at the point $o$. In this section we give an algorithm for locating a point $x_{a}$ in the interior of $\psi$ such that Area $\left\{\operatorname{VR}\left(x_{a}, S \cup\left\{\psi, x_{a}\right\}\right)\right\} \geq(1-\varepsilon) O P T$ Area, where $\varepsilon>0$. The techniques used in this section emulates the methods of Cheong et al. [9] for approximating the area of a Voronoi region a of new point, given a set of fixed points.

Suppose $S$ is a set of $n$ points placed inside $\psi$ and $x \notin S$ be another point which we wish to place inside $\psi$. Note that the function $\operatorname{Area}(\operatorname{VR}(x, S \cup\{x, \psi\}))$ is continuous in $x$. However, the limit of $\operatorname{Area}(\operatorname{VR}(x, S \cup\{x, \psi\}))$ as $x$ approaches a point $p_{i} \in S$ might not exist.

We define the reach of a Voronoi region $V R(x, S \cup\{x, \psi\})$ as the distance between the site $x$ and the furthest point inside $V R(x, S \cup\{x, \psi\})$. We denote the reach of a point $p_{i} \in S$ by $\ell_{i}$. The largest reach $\ell$ is the maximum of the reaches $\ell_{i}$, for $p_{i} \in S$.

Using this definition we now prove the following lower bound on OPTArea.

Lemma 4. OPTArea $\geq \pi \ell^{2} / 4$.

Proof. Let $x \notin S$ be a point in the interior of $\psi$ such that $\delta\left(x, p_{i}\right)=\ell$ for some $p_{i} \in S$. Then the disk $C$ centered at the point $x$ with radius $\ell$ contains no point of $S$ inside it. Moreover, since $x$ lies on the boundary of the Voronoi cell of $p_{i}, \delta(x, \psi) \geq \delta\left(x, p_{i}\right)$, Therefore, the disk $C$ is contained in $\psi$.

Next, we claim that the disk $D$ centered at $x$ with radius $\ell / 2$ is completely contained in $V R(x, S \cup\{x, \psi\})$. If not, there exists some point $y$ inside the disk $D$ with either $\delta\left(y, p_{j}\right)<$ $\delta(y, x)<\ell / 2$ for some $p_{j} \in S$, or $\delta(y, \psi)<\delta(y, x)<\ell / 2$. If the first case holds, then $\delta\left(x, p_{j}\right) \leq \delta(x, y)+\delta\left(p_{j}, y\right)<\ell$, which contradicts the emptiness of the disc $C$. Similarly, the second case follows.

Therefore, the disc $D$ is contained in $\operatorname{VR}(x, S \cup\{x, \psi\})$, which implies that OPT Area $\geq$ $\operatorname{Area}(\operatorname{VR}(x, S \cup\{x, \psi\})) \geq \pi \ell^{2} / 4$.

Note that the reach of $V R(x, S \cup\{x, \psi\})$ for any point $x \notin S$ is also at most $\ell$, which means that largest reach $\ell$ is the radius of the largest empty circle in $V(S \cup\{\psi\})$.

Let $Z$ be the square of side length 2 circumscribing the unit circle $\psi$. Now we partition $Z$ into a grid of squares with side length $\ell$. For each grid cell $Q$ in this partition, let $Q^{*}$ be the grid cell $Q$ clipped to the interior of the circle $\psi$. For a grid cell $Q$, we shall define an estimate function $E(x)$, such that for any $x \in Q^{*}$ we have,

$$
|E(x)-\operatorname{Area}(\operatorname{VR}(x, S \cup\{x, \psi\}))| \leq \frac{\varepsilon \pi \ell^{2}}{12} .
$$


Let $Q_{0}$ be a grid cell where $\sup _{x \in Q_{0}^{*}} \operatorname{Area}(\operatorname{VR}(x, S \cup\{x, \psi\}))=$ OPTArea, and let $x^{*} \in Q_{0}^{*}$ be such that point with $\operatorname{Area}\left(\operatorname{VR}\left(x^{*}, S \cup\left\{x^{*}, \psi\right\}\right)\right) \geq O P T$ Area $-\frac{\varepsilon \pi \ell^{2}}{12}$. For a grid cell $Q$, let $x_{Q}$ be the point which maximizes $E(x)$ over all points in $Q^{*}$, and $x_{a}$ be the point that maximizes $E\left(x_{Q}\right)$ over all grid cells $Q$. Then

$$
\begin{aligned}
\operatorname{Area}\left(\operatorname{VR}\left(x_{a}, S \cup\left\{x_{a}, \psi\right\}\right)\right) & \geq E\left(x_{a}\right)-\frac{\varepsilon \pi \ell^{2}}{12} \\
& \geq E\left(x^{*}\right)-\frac{\varepsilon \pi \ell^{2}}{12} \\
& \geq \operatorname{Area}\left(\operatorname{VR}\left(x^{*}, S \cup\left\{x^{*}, \psi\right\}\right)\right)-\frac{\varepsilon \pi \ell^{2}}{6} \\
& \geq \text { OPTArea }-\frac{\varepsilon \pi \ell^{2}}{4} \\
& \geq \text { OPTArea }-\varepsilon \text { OPT Area } \\
& =(1-\varepsilon) \text { OPTArea. }
\end{aligned}
$$

Now, we need to define $E(x)$ and describe a method to find the point $x_{Q}$, for each grid cell $Q$. We fix a grid cell $Q$, and let $x$ be a point in $Q^{*}$. The reach of $V R(x, S \cup\{x, \psi\})$ is at most $\ell$, and so $V R(x, S \cup\{x, \psi\})$ can intersect only $Q$ itself and its eight neighboring grid cells. Consequently, all points of $S$ participating in the definition of $V R(x, S \cup\{\psi\})$ lie in $Q$ and the 24 grid cells at distance at most $2 \ell$ from it. Let $Q^{\prime}$ denote the union of these 25 grid cells, and let $S_{Q}=S \cap Q^{\prime}$. We now make use of the following simple lemma.

Lemma 5. [9] Let $T$ be a square grid of density $\delta(>0)$ in the plane, that is, the distance between neighboring grid points is $\delta$, and let $\mathcal{C}$ be a convex body of diameter at most $\mathcal{D}$. Then $\left|\operatorname{Area}(\mathcal{C})-\delta^{2}\right| \mathcal{C} \cap T|| \leq 4 \mathcal{D} \delta$.

Let $\delta=\varepsilon \pi \ell / 96$ and $T$ be a square grid of density $\delta$ covering $Q^{\prime}$. For any point $x \in Q^{*}$ define,

$$
E(x)=\delta^{2}|V R(x, S \cup\{\psi\}) \cap T| .
$$

Making use of the fact that the diameter of $V R(x, S \cup\{x, \psi\})$ is at most $2 \ell$, we have by Lemma 5 ,

$$
|E(x)-V R(x, S \cup\{x, \psi\})| \leq 8 \ell \delta=\frac{\varepsilon \pi d^{2}}{12} .
$$

Now, to find the point $x_{Q}$ which maximizes $E(x)$ for $x \in Q^{*}$ we need the following lemma.

Lemma 6. If $W(t)=\left\{x \in Q^{*} \mid t \in V R(x, S \cup\{x, \psi\})\right\}$, then $W(t)$ is the largest disc with center at $t$ containing no point of $S$, which is clipped to $Q^{*}$ and contained in $\psi$.

Proof. Clearly $W(t)=\bigcap_{i=1}^{p}\left\{x \in Q^{*} \mid t \in V R\left(x,\left\{x, p_{i}\right\}\right)\right\} \bigcap\left\{x \in Q^{*} \mid t \in V R(x,\{x, \psi\})\right\}$. For every $p_{i} \in S,\left\{x \in Q^{*} \mid t \in V R\left(x,\left\{x, p_{i}\right\}\right)\right\}$ is the largest disk with center at $t$ not containing the point $p_{i}$, clipped to $Q^{*}$. The result now follows from the observation that $\left\{x \in Q^{*} \mid t \in V R(x,\{x, \psi\})\right\}$ is the disk with center at the point $t$ and radius $\delta\left(t, t_{\psi}\right)$, clipped to $Q^{*}$, where $t_{\psi}$ is the point on the circumference of $\psi$ which is nearest to $t$. 
Let $\mathcal{W}_{T}=\{W(t) \mid t \in T\}$ and consider the arrangement $\mathcal{A}\left(\mathcal{W}_{T}\right)$. From Lemma 6 , the problem of finding the point $x_{Q}$ now reduces to finding a point in $Q^{*}$ that is contained in the largest number of clipped discs in $\mathcal{W}_{T}$.

Since the Voronoi diagram $V(S \cup\{\psi\})$ and the largest reach $\ell$ can be computed in $O(n \log n)$ time [17], the next theorem follows from arguments exactly similar to those in Theorem 3.3 of Cheong et al. [9].

Theorem 5. Given a set $S$ of $n$ points in the plane and a parameter $\varepsilon>0$, one can deterministically compute, in time $O\left(n / \varepsilon^{4}+n \log n\right)$, a point $x_{a}$ such that Area $\left(\operatorname{VR}\left(x_{a}, S \cup\right.\right.$ $\left.\left.\left\{x_{a}, \psi\right\}\right)\right) \geq(1-\varepsilon)$ OPT Area.

Proof. We start by computing the Voronoi diagram of $S \cup\{\psi\}$ and determine the largest reach $\ell$, which takes $O(n \log n)$ time [17]. We then define the square grid, and determine the set of points $S_{Q}$ relevant in each grid cell. Since a point of $S$ is relevant in at most 25 grid cells, the total size of the sets $S_{Q}$ is $O(n)$.

For each grid cell $Q$ we take a square grid $T$ of density $\delta$. It consists of $M=O\left(1 / \varepsilon^{2}\right)$ points. For $t \in T$, the clipped disc $W(t)$ can be determined by finding the nearest neighbor to $t$ in $S_{Q} \cup\left\{t_{\psi}\right\}$. This can be done by comparing the distance from $t$ to each point in $S_{Q} \cup\left\{t_{\psi}\right\}$. The arrangement $\mathcal{W}_{T}$ is computed by a sweep-line algorithm in time $O\left(M^{2}\right)$, and we pick a point $x_{Q}$ from the face maximizing the estimate $E\left(x_{Q}\right)$ by a simple traversal.

By the choice of $\ell$, every grid cell is within distance at most $2 \ell$ from a point of $S$. The number of grid cells handled is therefore at most $O(n)$. Each point of $S$ appears at most $O(M)$ times in a nearest-neighbor computation, and so the overall running time is $O\left(n \log n+n M+n M^{2}\right)=O\left(n / \varepsilon^{4}+n \log n\right)$.

Following the proof of Theorem 5 , it is now easy to formulate a $(1-\varepsilon)$-approximation algorithm for problem $\mathrm{P} 2$, which runs in $O\left(n / \varepsilon^{4}+n \log n\right)$ time.

\section{Conclusions}

In this paper, we considered two problems related to maximizing the area of Voronoi regions of a circle and a set of $n$ points placed inside it. These are motivated from various applications in facility location and disaster management problems, where both stationary and mobile service stations are deployed. The interior of the circle is partitioned into the Voronoi region of the points and the Voronoi region of the circle itself. In the first problem, the objective is to place the points such that the total area of the Voronoi region of these points is maximized. We considered a restricted scenario where the distances of each of these points from the center of the circle are the same. In this case, the optimum solution is obtained when these points lie on the vertices of a regular $n$-gon. The general problem is still open. However, we conjecture that even in the general case, the optimal solution is identical to the solution of the above-mentioned restricted problem. The rationale behind this conjecture is the observation that the optimum is likely to be attained when the areas of the service zones of all the stationary facilities become equal. Therefore, the special case where the stationary facilities are assumed to be equidistant from the center of the circle, is likely to provide the optimum solution for the general case as well.

In the second problem, a circle enclosing a set of $n$ points is given, and the objective is to place a new point such that its Voronoi region is maximized. We provide an exact solution to this problem for $n=1$, and propose an approximation algorithm for the general case.

It would be interesting to study similar maximization problems when the outer circle is replaced by other bounding shapes, for example a convex polygon. Solutions of these 
problems using other related distance functions are also of theoretical interest.

Acknowledgement: The author wishes to thank Professors Probal Chaudhuri, Sandip Das, and Subhas C. Nandy of the Indian Statistical Institute, Kolkata, and Professor Rolf Klein of the Institut für Informatik I, Universität Bonn, Germany, for their insightful suggestions. The author is also grateful to the two anonymous referees for their critical comments, which have greatly improved the quality of the paper.

\section{References}

1. H. K. Ahn, S. W. Cheng, O. Cheong, M. Golin, and R. van Oostrum, Competitive facility location along a highway, 7th Annual International Computing and Combinatorics Conference, LNCS Vol. 2108, 237-246, 2001.

2. H. Alt, O. Cheong, and A. Vigneron, The Voronoi diagram of curved objects, Discrete and Computational Geometry, Vol. 34, 439-453, 2005.

3. T. Apostol, Mathematical Analysis, Narosa Publishing House, reprinted 2002.

4. F. Aurenhammer, Voronoi Diagrams - A Survey of a Fundamental Geometric Data Structure, ACM Computing Surveys, Vol. 23, No. 3, September 1991.

5. F. Aurenhammer and R. Klein, Voronoi diagrams: Handbook of Computational Geometry, Elsevier, Amsterdam, 2000.

6. B. B. Bhattacharya and S. C. Nandy, New variations of the reverse facility location problem, Proc. 22nd Canadian Conference on Computational Geometry, to appear, 2010.

7. M. de Berg, M. van Kreveld, M. Overmars, and O. Schwarzkopf, Computational Geometry: Algorithms and Applications, 2nd ed. New York: Springer-Verlag, May 2000.

8. S. Cabello, J. Miguel Diáz-Báñez, S. Langerman, C. Seara, and I. Ventura, Facility location problems in the plane based on reverse nearest neighbor queries, European Journal of Operations Research, Vol. 202(1), 99-106, 2010.

9. O. Cheong, A. Efrat, and S. Har-Peled, On finding a guard that sees most and a shop that sells most, Discrete and Computational Geometry, Vol. 37(4),545-563, 2007.

10. O. Cheong, S. Har-Peled, N. Linial, and J. Matoušek, The one-round Voronoi game, Discrete and Computatinal Geometry and Applications, Vol. 31(1), 125-138, 2004.

11. F. Dehne, R. Klein, and R. Seidel, Maximizing a Voronoi region: The convex case, International Journal of Computational Geometry and Applications, Vol. 15(5), 463-475, 2005.

12. Z. Drezner and H. W. Hamacher (Eds.), Facility Location: Applications and Theory, Springer, 2002.

13. I. S. Gradshteyn and I. M. Ryzhik, Tables of Integrals, Series, and Products, Academic Press, Orlando, 1980.

14. S. L. Loney, Elements of Coordinate Geometry, Macmillan and Co., reprinted 1953.

15. A. Okabe, B. Boots, and K. Sugihara, Spatial Tessellations: Concepts and Applications of Voronoi Diagrams, Wiley, 1992.

16. Voronoi Diagrams of points, segments, and arcs in 2D; http://www.cosy.sbg.ac.at/ held/projects/vroni/.

17. C. K. Yap, An $O(n \log n)$ time algorithm for the Voronoi diagram of a set of simple curve segments, Discrete and Computational Geometry, Vol. 2, 365-393, 1987. 